\title{
Sommerflor im Palmengarten
}

Hilke Steinecke

Jedes Jahr neu ist im Palmengarten an verschiedenen Stellen ein farbenfroher Sommerflor zu bestaunen. Verwendet werden überwiegend einjährige Pflanzen, die in der Gärtnerei des Palmengartens herangezogen werden. Mitte Mai, also nach den Eisheiligen, werden sie ausgepflanzt und erfreuen mit ihrer Blütenpracht bis zum Herbst, wenn dann die Sommerbepflanzung abgeräumt wird. Anschließend werden die Beete für den Winter vorbereitet. Im Laufe des Sommers verändert sich das Bild, da nach und nach unterschiedliche Pflanzen ihre Hauptblüte entwickeln und gegen Ende der Saison größer werdende Exemplare dominieren, während die kleineren zurückgedrängt werden.

Regelmäßig werden die vier Beete um das Wasserbecken am Eingang Siesmayerstraße mit einer Wechselbepflanzung ausgestattet. Im Sommer 2014 dominierten hier vier Bananenstauden, die schon dem Eingangsbereich ein tropisches Ambiente verliehen. Neu in diesem Sommer war auch die Verwendung eines aus Nordamerika stammenden Wasserdostes (Eupatorium capillifolium 'Elegant Feather'). Die in Amerika auch dog fennel (Hunde-Fenchel) genannte Staude hat einen aufrechten Wuchs. Sie wird $2 \mathrm{~m}$ hoch und verzweigt sich an ihrer Basis. Mit ihren fadenförmig geteilten Blättern wirkt sie sehr zierlich; sie ist deshalb ein beliebtes gestalterisches Element. Da die Staude etwas

Abb. 1: Sommerflor am Eingangsschauhaus: Bananenstaude und Eupatorium capillifolium 'Elegant Feather' prägen das Bild.

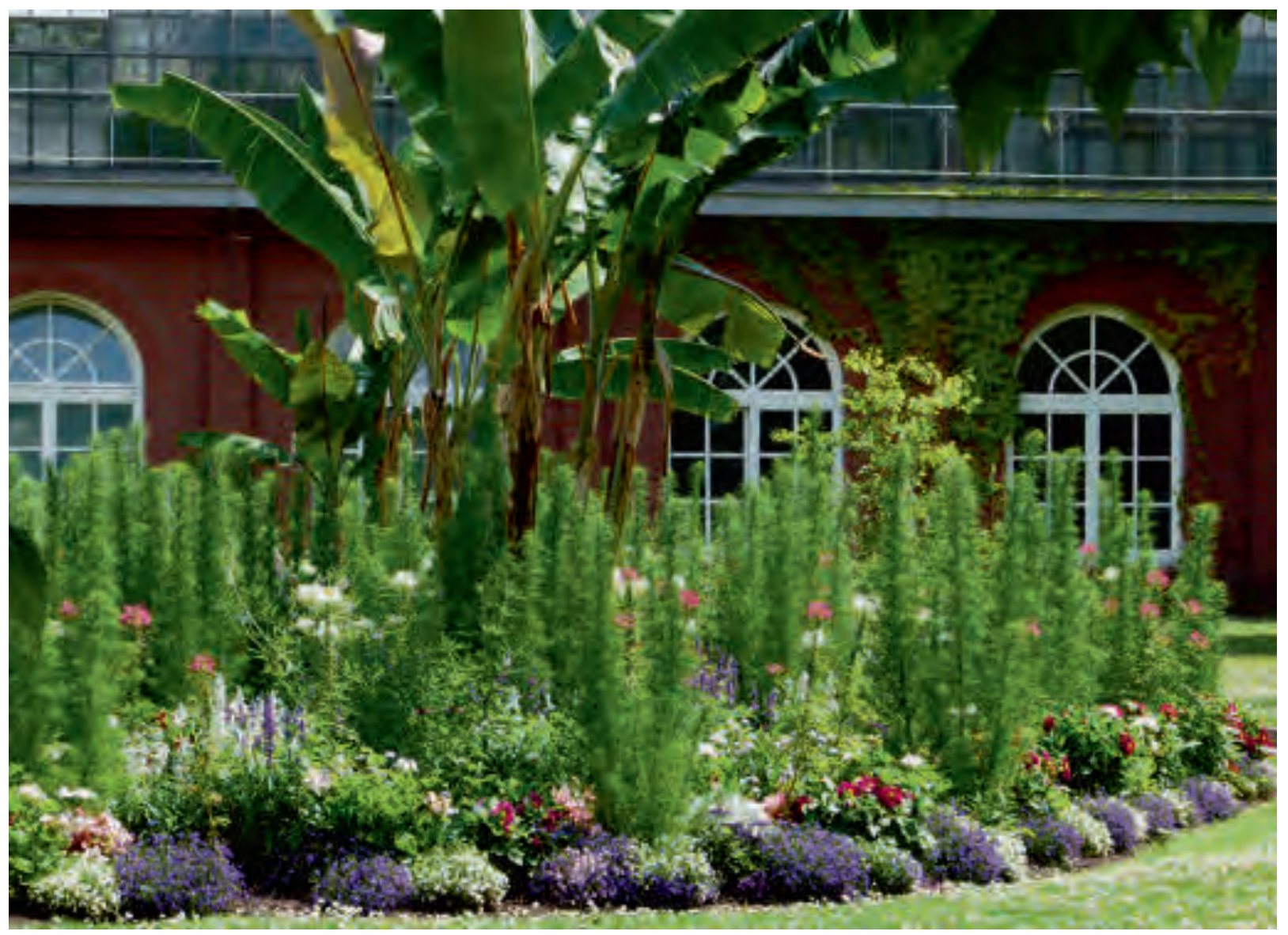




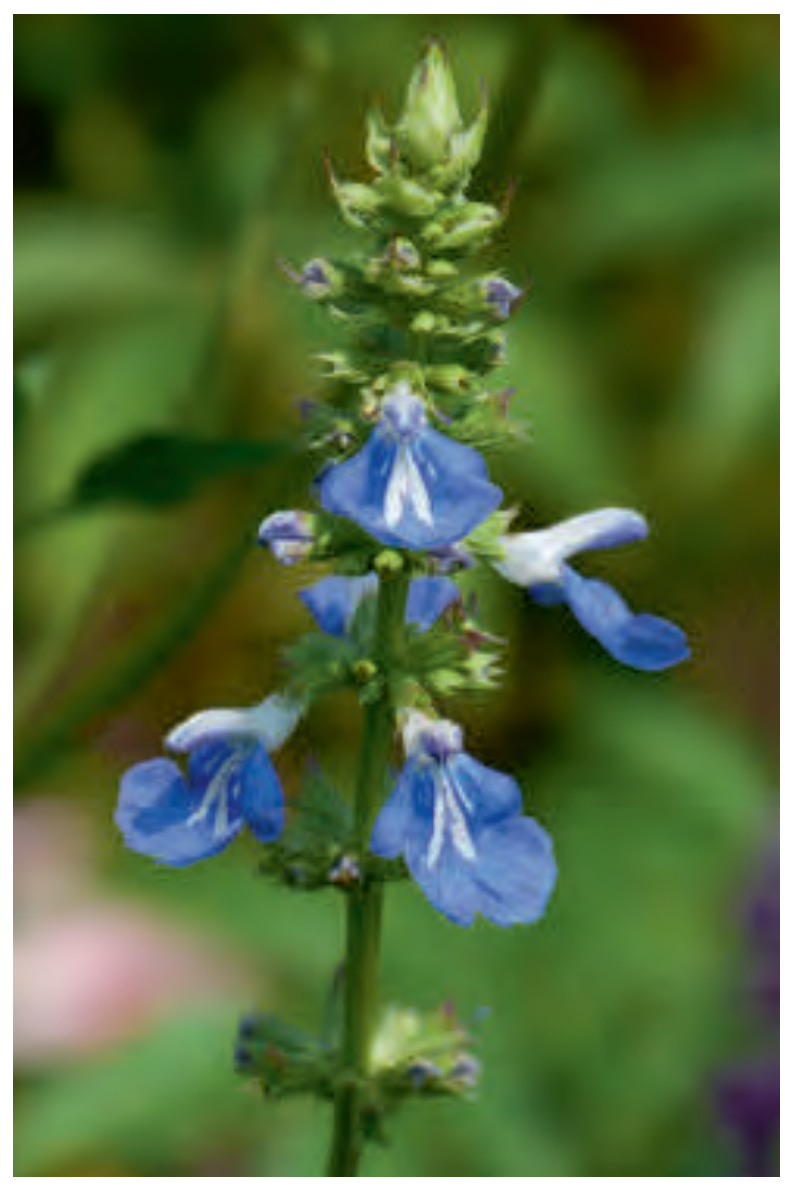

an einen kleinen Nadelbaum erinnert, wird sie gelegentlich auch Sommer-Zeder genannt. Den Blättern entströmt beim Zerreiben ein etwas strenger, aromatischer Duft (daher die weitere Bezeichnung stinking chamomile, Stink-Kamille). Zum Würzen sind die Blätter nicht geeignet, denn sie enthalten Pyrrolizidin-Alkaloide, die sich schädlich auf die Leber auswirken. Bei äußerlicher Anwendung sind sie als Mücken-Repellent zu verwenden. Die kleinen unscheinbaren Blütenköpfchen sind weiß bis zart rosa gefärbt. In warmen Regionen kann sich dog fennel schnell an Straßenrändern oder auf Brachland vermehren und ist gebietsweise ein invasiver Neophyt. Da die Staude unter unseren klimatischen Verhältnissen keinen Samen ausbildet, ist die Gefahr der unkontrollierten Ausbreitung bei uns nicht gegeben.

Bis zum Herbst hält sich in unserem Sommerflor sehr gut der Pfeffer-Salbei (Hummelschaukel, Salvia uliginosa) mit seinen himmelblauen Blüten. Dieser in Südamerika heimische Salbei wächst bevorzugt auf sonnigen, feuchten
Wiesen. Er gedeiht aber auch auf normalen, nährstoffreichen, nicht zu trockenen Böden und ist bei uns als Kübelpflanze geeignet, denn Fröste unter $-10^{\circ} \mathrm{C}$ verträgt er nicht. PfefferSalbei blüht vom Hochsommer bis zum Oktober und erreicht dann Höhen bis $2 \mathrm{~m}$. Gewöhnlich wächst dieser Salbei zu dichten, buschigen Stauden heran. Sie sind eine gute Bienen- bzw. Hummelweide. Wenn dicke, relativ schwere Hummeln die Blüten aufsuchen, gerät gelegentlich der lange Blütenstand ins Schwanken, daher der deutsche Name Hummelschaukel. Die Blüten sind essbar und eignen sich hervorragend für Salate und Süßspeisen. Die Blätter haben einen pfeffrig-scharfen Geschmack.

Seit einigen Jahren zeigen wir längs des Tropicariums eine farbenfrohe Millefleurs-Pflanzung, die durch ihre Leichtigkeit wirkt. Millefleurs-Pflanzungen haben ihren Ursprung als sogenannte „Emaille-Pflanzung“ in der späten Barockzeit sowie im Rokkoko. In diese Pflanzungen wurden früher gerne neue und besonders kostbare Pflanzen integriert. Von vielen Neuheiten war oft nur wenig über Habitus und Blütezeit bekannt, sodass sich im Laufe des Jahres in der Pflanzung gelegentlich ein Durcheinander entwickelte, das aber auch seine besonderen Reize hatte. Im 20. Jh. gerieten Millefleurs-Pflanzungen außer Mode. Seit den 1980er Jahren sind sie in Deutschland wieder deutlich beliebter geworden. Neben der farblichen Abstimmung kennzeichnet eine solche Pflanzung die sorgfältige Höhenstaffelung zwischen etwa 20 und $80 \mathrm{~cm}$, wobei die höher wachsenden Pflanzen in lockeren kleinen Gruppen zusammenstehen sollten. So wirkt das Ensemble wie ein riesiger bunter Blumenstrauß. Farbe und Form der Blüten müssen sich von der Waagerechten zur Senkrechten vom Schweren

Abb. 2 (oben): Salvia uliginosa wird erst gegen Ende der Vegetationsperiode besonders kräftig.

Abb. 3 (Seite 77 oben): Neben den Washingtonien am Wasserbecken wurden Kübel mit Agapanthus aufgestellt.

Abb. 4 (Seite 77 unten): Sommerflor entlang des Tropicariums, im Hintergrund hohe Sonnenblumen mit schwarzbraunen Blütenköpfen. 

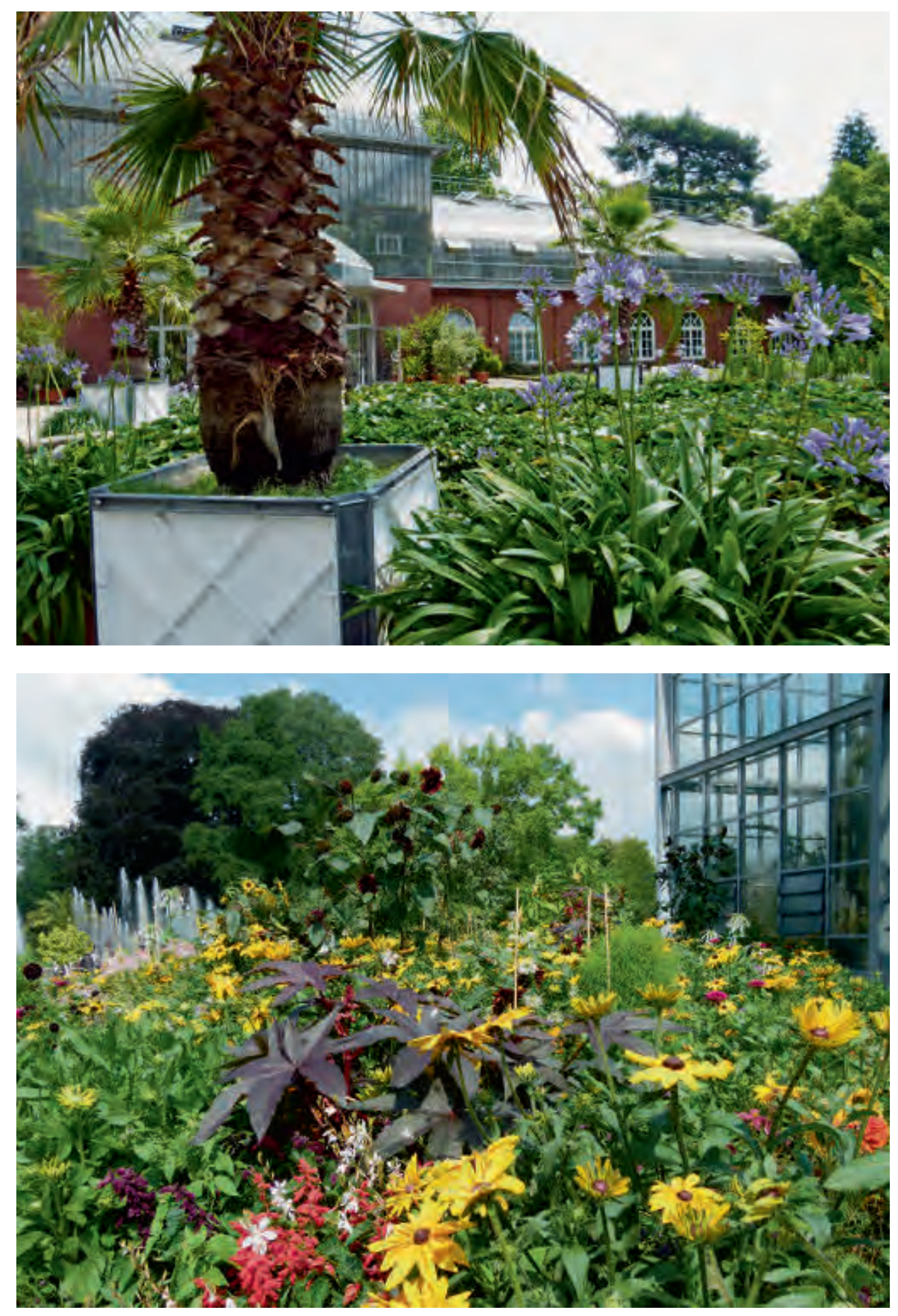


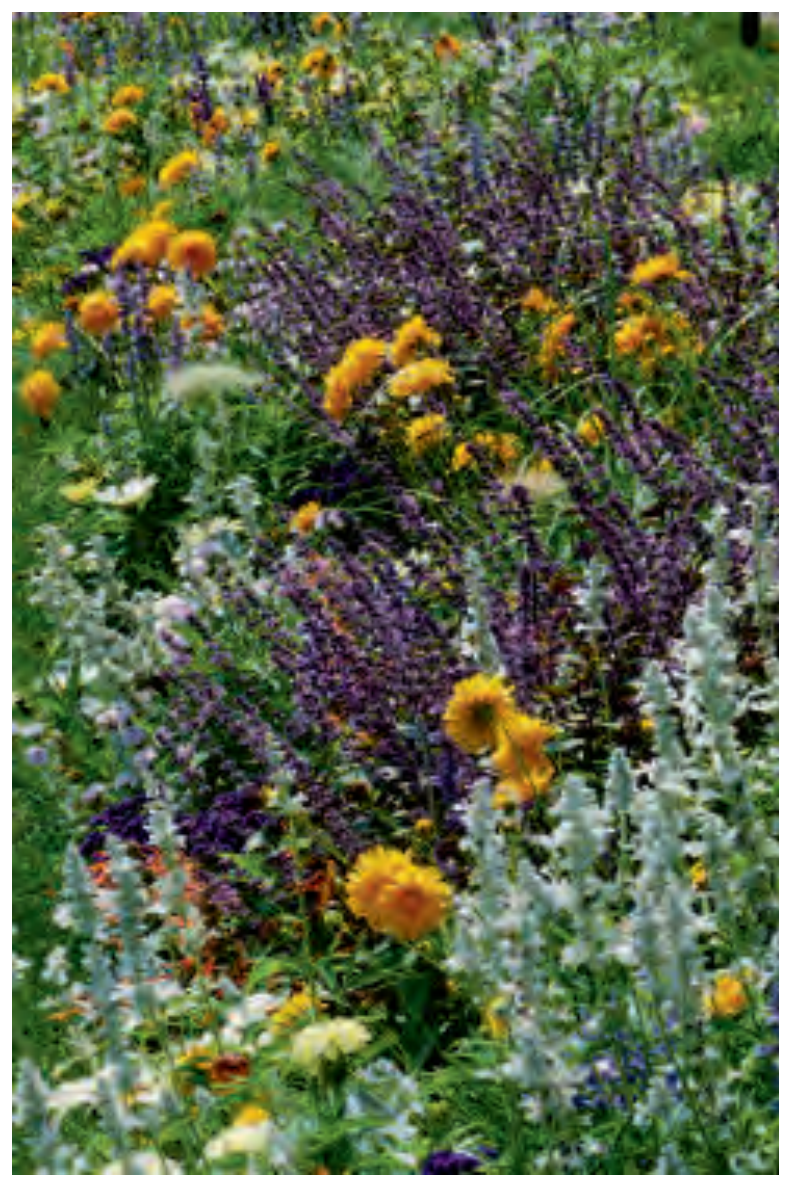

(dunkle Farben) zum Leichten (helle Farben) entwickeln. Sehr gut geeignet als halbhoher Partner zu den niedrigen Teppichpflanzen sind Cosmos bipinnatus oder Zier-Tabak (z. B. Nicotiana sylvestris). Letzterer wird jedes Jahr in unserem Sommerflor verwendet. Seine engröhrigen, weißen Blüten duften am Abend und locken Nachtfalter an, die mit ihrem langen Rüssel an den Nektar im Blütengrund gelangen und dabei als Bestäuber fungieren.

Die Kunst einer guten Millefleurs-Pflanzung besteht darin, dass sie mit einem gewissen jahreszeitlichen Wechsel während der gesamten Sommersaison blüht. Sehr beruhigend zwischen den bunten Blüten wirken verschiedene Blattpflanzen, darunter Greiskraut (Senecio) mit silbergrauen Blättern oder Gräser (z. B. Pennisetum). Es wirkt sehr gut, wenn sich im Zentrum des Beetes mittelhohe und hohe Blumen in der Kontrastfarbe der Kanten entwickeln können. In diesen herausragenden „Sträußen“ sollten bei entsprechendem Platzangebot nicht Einzelpflanzen, sondern 3-5 Pflanzen einer
Sorte zusammenstehen. Innerhalb der Pflanzung können sich straußartige Gruppen wiederholen. Ab Juli 2014 prägten verschiedene Sorten der Einjährigen Sonnenblume ('Peach Passion', 'Moulin Rouge', 'Goldmine', 'Pro Cut Orange') das Bild.

Die Sommerbepflanzung 2014 des Blumenparterres vor dem Gesellschaftshaus war durch Leichtigkeit und Wildblumencharakter geprägt. Außerdem wurde die Thematik „Duft", passend zur großen Jahresausstellung, mit aufgenommen. Für die Gestaltung der Beete wurden deshalb verschiedene Duft- und Gewürzpflanzen wie z. B. Salvia officinalis 'Berggarten', Ocimum x hybridum ('La Palma', 'Gusto Azorro', Gusto Bianco'), Heliotropium arborescens 'Mini Marine' oder Agastache aurantiaca 'Apricot Sprite' verwendet. Während im vergangenen Jahr kräftige Gelb-Orange-Rot-Töne dominierten, waren diesmal zartere Farben und Crème-Töne an der Reihe. Zur Auflockerung wurden die Gräser Pennisetum villosum 'Nemira' und Nasella (= Stipa) tenuissima ('Ponytails') gepflanzt. Passend zur weißen Fassade des Gesellschaftshauses war die Pflanzung auf dem Hangbeet luftig weiß gehalten. Lavatera trimestris 'Mont Blanc', Anthirrinum majus 'Rocket White', Orlaya grandiflora 'White Lace' und einige andere Pflanzen mit weißen Blüten oder silbriggrauem Laub wurden ab dem Hochsommer durch einen hoch wachsenden Fenchel (Foeniculum vulgare) mit rötlichbraunen Stängeln ergänzt bzw. abgelöst.

Abb. 5 (oben): Duftende Randbepflanzung des Blumenparterres.

Abb. 6 (Seite 79 oben): Blumenparterre mit Gesellschaftshaus.

Abb. 7 (Seite 79 unten): Hangbeet mit BuschMalven und Fenchel. 

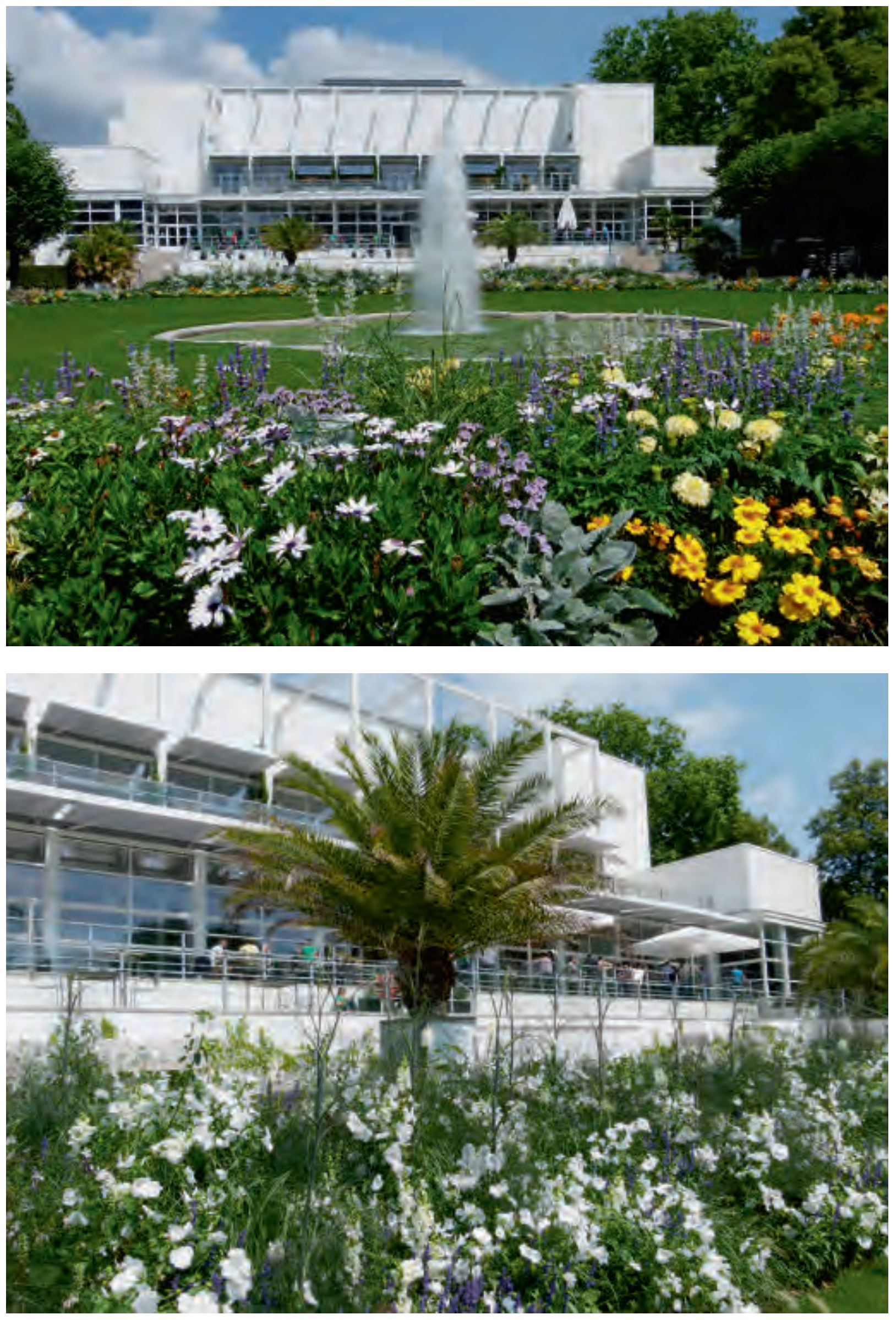